\title{
Estimation of stable CARMA models with an application to electricity spot prices
}

\author{
Isabel García* Claudia Klüppelberg ${ }^{\dagger} \quad$ Gernot Müller ${ }^{\ddagger}$
}

January 25, 2010

\begin{abstract}
We discuss theoretical properties and estimation of continuous-time ARMA (CARMA) processes, which are driven by a stable Lévy process. Such processes are very useful in a continuous-time linear stationary set-up: they have a similar structure as the widely used ARMA models, and provide all advantages of a continuous-time model. As an application we consider data from a deregulated electricity market. Here we fit a CARMA $(2,1)$ model to spot prices from the Singapore New Electricity Market. The quality of the estimates is assessed in a simulation study. The continuous-time modelling aims at a new pricing methodology for energy derivatives.
\end{abstract}

AMS 2010 Subject Classifications: primary: 62M10, 60G52, $91 \mathrm{G} 70$

secondary: $62 \mathrm{M} 05$

Keywords: CARMA model, electricity prices, estimation of CARMA models, stable CARMA model, stable Ornstein-Uhlenbeck process, stable Lévy process.

\footnotetext{
*Department of Mathematics, Ruhr-Universität Bochum.

${ }^{\dagger}$ Center for Mathematical Sciences, and Institute for Advanced Study, Technische Universität München, D-85747 Garching, Germany, Email: cklu@ma.tum.de, URL: http://www-m4.ma.tum.de

${ }^{\ddagger}$ Center for Mathematical Sciences, Technische Universität München, D-85747 Garching, Germany, Email: mueller@ma.tum.de, URL: http://www-m4.ma.tum.de/pers/mueller/
} 


\section{Introduction}

Since the discovery of the light bulb, electricity has made a tremendous impact on the development of our society. Today, it represents a crucial component of modern way of life, and it is hard to imagine a life without it.

In recent years, electricity markets throughout Europe have undergone massive changes due to deregulations. The original monopolistic situation was replaced by deregulated markets, where consumers in principle are free to choose their provider so that the market for electric power has become competitive. Before, most power sectors were not exposed to competition, and prices were set by regulators according to the cost of generation, transmission and distribution. Accordingly, hardly any price risks existed. On spot markets of energy exchanges, where electricity for delivery on the next day is traded, the situation is quite different. Here prices are determined purely by supply and demand, and hence they fluctuate considerably. As a consequence, utilities, distributors and industrial companies are faced with electricity price risks. Knowledge about the stochastic properties of prices and risk premiums - which are paid to ensure against these price risks are essential for a successful risk management.

A main characteristic for electricity is its very limited storability. It is hardly possible to ensure against price risks by building reserves. As a consequence, a sudden rise in electricity demand (e.g. due to a failure of a power plant) often results in significant price jumps which are observed frequently in most electricity markets. These are called price spikes and are unique to electricity markets, such that standard models for commodities do not describe observed prices in a realistic way.

With deregulation and the introduction of competition a new challenge for power market participants has emerged. Extreme price volatility, which can be even two orders of magnitude higher than for other commodities or financial instruments, has forced producers and wholesale consumers to hedge not only against volume risk but also against price movements. Price forecasts have become a fundamental input to an energy company's decision-making and strategy development. This turn has fostered research in electricity price modelling and forecasting.

In conclusion, statistical modeling and estimation of electricity spot prices are an important issue with consequences in pricing of energy derivatives and risk management of electricity markets. The data exhibit certain features of commodity data as well as financial data. So-called stylized facts present some universal characteristics of 
electricity spot price data; for details we refer to Bernhardt, Klüppelberg and MeyerBrandis [1] and Weron [22]. Obviously, such data exhibit yearly, weekly and daily seasonality. In contrast to most financial data, electricity spot prices are stationary (after removal of the seasonal effects). Electricity spot prices show impressive spikes and strong leptokurtic behaviour, indeed second moments do not exist.

In Klüppelberg, Meyer-Brandis and Schmidt [13] a three-factor continuous-time model was suggested for electricity spot prices, which has been used for pricing of electricity derivatives in Meyer-Brandis and Tankov [15]. Although the model captures the stylized features very well, it is somewhat hard to fit statistically. On the other hand, in Bernhardt et al. [1] a discrete time stable ARMA model has been suggested, which also captured the stylized features very well, and, as a fairly standard linear model, is easy to fit, although the driving noise is stable. This means that no finite variance exists, so that the usual Hilbert space arguments do not apply. It has been shown, on the other hand, that standard $L^{2}$-estimation procedures can be applied also in this heavy-tailed setup; cf. Davis [8] and Mikosch et al. [16] and references therein.

In the present paper we extend the simple model used in Bernhardt et al. [1] to a continuous-time setup. Our long term vision aims at pricing of energy derivatives for which a continuous-time model is preferable.

So-called continuous ARMA (CARMA) models have been suggested already by Doob [9] and rediscovered by Brockwell [3], so that an extensive literature exists by now, mainly concentrated on finite variance models. Although at first sight it may seem straighforward to define a CARMA process and apply statistical tools developed for ARMA processes to data, which are necessarily sampled at discrete times, this is not so straightforward. We want to mention some difficulties one has to deal with.

- Whereas there exist stationary and causal $\operatorname{ARMA}(p, q)$ processes for every $p, q \geq$ $1, \operatorname{CARMA}(p, q)$ processes exist for $q<p$; see Brockwell [3].

- Estimators usually exhibit a different behavior for ARMA and CARMA models (e.g. in terms of asymptotic properties etc.), although for a very fine grid there are similarities; cf. Brockwell [2], Section 6.

- For many applications it is important to estimate the driving Lévy process. This is possible, but not straightforward; cf. Brockwell, Davis and Yang [5]. 
Our paper is organised as follows. In Section 2 we present some theoretical results on CARMA processes with special emphasis on the driving stable Lévy process. Section 3 is devoted to the statistical estimation procedure. In Section 4 we conduct a simulation study to check our estimation procedure, and in Section 5 we analyze the Singapore electricity spot price data using a stable CARMA model. Section 6 concludes.

\section{Stable CARMA processes}

Throughout we work on a filtered probability space satisfying the usual conditions of completeness and right-continuity. Let $\{L(t)\}_{t \geq 0}$ be an $\alpha$-stable Lévy process; i.e.

- $L(0)=0$ a.s.

- $L$ has independent increments.

- For every $0 \leq s<t<\infty$ the random variable

$$
L(t)-L(s) \sim S_{\alpha}\left(c(t-s)^{1 / \alpha}, \beta, \mu\right)
$$

for some $\alpha \in(0,2), \beta \in[-1,1], c>0, \mu \in \mathbb{R}$, i.e. the characteristic function has for all $t \geq 0$ the representation $\mathbb{E}\left[e^{i \theta L(t)}\right]=e^{t \xi(\theta)}$ for $\theta \in \mathbb{R}$, where (cf. Definition 1.1.6 of Samorodnitsky and Taqqu [18])

$$
\xi(\theta)=\left\{\begin{array}{c}
-c^{\alpha}|\theta|^{\alpha}\left(1-i \beta(\operatorname{sign} \theta) \tan \left(\frac{\pi \alpha}{2}\right)\right)+i \mu \theta \quad \text { for } \alpha \neq 1 \\
-c|\theta|\left(1+i \beta \frac{2}{\pi}(\operatorname{sign} \theta) \log |\theta|\right)+i \mu \theta \quad \text { for } \alpha=1
\end{array}\right.
$$

The sign function $\operatorname{sign} \theta=-1,0,1$ according as $\theta<0, \theta=0$ or $\theta>0$, respectively.

If $c=1$ and $\mu=0$, then $L$ is called standardized.

- $L$ has càdlàg sample paths.

W.l.o.g. we assume $\mu=0$, since it affects only location and can be subtracted, if needed, cf. Property 1.2.6 and Corollary 1.2.7 in Samorodnitsky and Taqqu [18].

A Lévy-driven $\operatorname{CARMA}(p, q)$ process $\{Y(t)\}_{t \geq 0}$ with $p, q \in \mathbb{N}_{0}$ such that $q<p$ and coefficients $a_{1}, \ldots, a_{p}, b_{0}, \ldots, b_{q} \in \mathbb{R}$ is defined via the state-space representation of the formal equation

$$
a(D) Y(t)=b(D) D L(t), \quad t \geq 0,
$$


where $D$ denotes differentiation with respect to $t$ (see below), $\{L(t)\}_{t \geq 0}$ is a stable Lévy process as defined above,

$$
a(z):=z^{p}+a_{1} z^{p-1}+\cdots+a_{p} \quad \text { and } \quad b(z):=b_{0}+b_{1} z+\cdots+b_{p-1} z^{p-1}
$$

are the characteristic polynomials, and the coefficients $b_{j}$ satisfy $b_{q}=1$ and $b_{j}=0$ for $q<j<p$.

Since the derivative $D L(t)$ does not exist in the usual sense, we interpret (2.2) as being equivalent to the observation and state equations

$$
\begin{gathered}
Y(t)=\mathbf{b}^{T} \mathbf{X}(t) \\
d \mathbf{X}(t)=A \mathbf{X}(t) d t+\mathbf{1}_{p} d L(t),
\end{gathered}
$$

where

$$
\begin{gathered}
\mathbf{X}(t)=\left(\begin{array}{c}
X(t) \\
X^{(1)}(t) \\
\vdots \\
X^{(p-2)}(t) \\
X^{(p-1)}(t)
\end{array}\right), \quad \mathbf{b}=\left(\begin{array}{c}
b_{0} \\
b_{1} \\
\vdots \\
b_{p-2} \\
b_{p-1}
\end{array}\right), \quad \mathbf{1}_{p}=\left(\begin{array}{c}
0 \\
0 \\
\vdots \\
0 \\
1
\end{array}\right), \\
A=\left(\begin{array}{ccccc}
0 & 1 & 0 & \ldots & 0 \\
0 & 0 & 1 & \ldots & 0 \\
\vdots & \vdots & \vdots & \ddots & \vdots \\
0 & 0 & 0 & \ldots & 1 \\
-a_{p}-a_{p-1}-a_{p-2} & \ldots & -a_{1}
\end{array}\right) \text { and } A=-a_{1} \text { for } p=1 .
\end{gathered}
$$

State space representations of continuous-time AR and ARMA processes date back to Brockwell, Hyndman and Grunwald [6]. For $(p, q)=(1,0)$ and $(p, q)=(2,1)$, the equivalence of $(2.2)$ and $(2.3) /(2.4)$ is illustrated in Tsai and Chan [19], pp. 585-586. Equation (2.4) is the short notation for the stochastic integral equation

$$
\mathbf{X}(t)=e^{A(t-s)} \mathbf{X}(s)+\int_{(s, t]} e^{A(t-u)} \mathbf{1}_{p} d L(u), \quad 0 \leq s<t,
$$

where the stable integral is defined as in Chapter 3 of Samorodnitsky and Taqqu [18]. Such infinite variance models have already been mentioned in Brockwell [3].

Remark 2.1. The eigenvalues of the matrix $A$, which we shall denote by $\lambda_{1}, \lambda_{2}, \ldots, \lambda_{p}$, are the same as the zeros of the autoregressive polynomial $a(\cdot)$, which can easily 
be verified by expanding the determinant of $\lambda I-A$ along the first column using Laplace's formula. The corresponding right eigenvectors are $\left(1, \lambda_{i}, \lambda_{i}^{2}, \ldots, \lambda_{i}^{p-1}\right)^{T}$ for $i=1, \ldots, p$. Note that these vectors are always eigenvectors, even in the case of not pairwise distinct eigenvalues.

The following result guarantees the existence of a strictly stationary solution $\mathbf{X}$ of (2.5). In order to formulate it we have to extend the stochastic integral in (2.5) to the negative half-line. To extend the Lévy process $L$ to the whole real line we take two independent copies $L_{1}$ and $L_{2}$ of $L$ and define

$$
L(t)=L_{1}(t) I_{[0, \infty)}(t)-L_{2}(-t-) I_{(-\infty, 0)}(t)
$$

Proposition 2.2 (cf. Brockwell and Lindner [7], Theorem 3.3, Brockwell et al. [5], Proposition 1).

(a) Let $L$ be a Lévy process (non-deterministic) and assume that $a(\cdot)$ and $b(\cdot)$ have no common zeros. Then the CARMA equations (2.3) and (2.4) have a strictly stationary solution $Y$ on $\mathbb{R}$ if and only if $E \log ^{+}\left|L_{1}\right|<\infty$ and $a(\cdot)$ is non-zero on the imaginary axis. In this case the solution $\{Y(t)\}_{t \in \mathbb{Z}}$ is unique and the corresponding state vector process $\{\mathbf{X}(t)\}_{t \in \mathbb{R}}$ can be chosen to be strictly stationary.

(b) There exists a strictly stationary solution $\mathbf{X}$ of (2.5) with the property that $\mathbf{X}(t)$ is independent of $\{L(s)-L(t), s>t\}$ for all $t \in \mathbb{R}$ if and only if the conditions in (a) hold and that all eigenvalues of $A$ have negative real parts:

$$
\Re\left(\lambda_{i}\right)<0, \quad i=1, \ldots, p .
$$

This solution has representation

$$
\mathbf{X}(t)=\int_{(-\infty, t]} e^{A(t-u)} \mathbf{1}_{p} d L(u), \quad t \in \mathbb{R},
$$

which has for all $t \in \mathbb{R}$ the same distribution as $\int_{0}^{\infty} e^{A u} \mathbf{1}_{p} d L(u)$.

(c) Assume that the conditions in (a) and (2.6) hold. Then

$$
Y(t)=\mathbf{b}^{T} \mathbf{X}(t)=\int_{(-\infty, t]} g(t-u) d L(u), \quad t \in \mathbb{R}
$$

where $g(h)=\mathbf{b}^{T} e^{A h} \mathbf{1}_{p}$ for $h \geq 0$ is referred to as the kernel of $\{Y(t)\}_{t \in \mathbb{R}}$. The process $Y$ is causal in the sense that $Y(t)$ is independent and $\{L(s), s>t\}$ for all $t \in \mathbb{R}$. 
The following result specifies the finite dimensional distributions of the $\operatorname{CARMA}(p, q)$ process. It has been proved in Theorem 2.2 of Brockwell [3] under the assumption of finite second moments, which was also used in the proof. For this reason we give a short proof for our situation.

\section{Proposition 2.3.}

If $\{L(t)\}_{t \in \mathbb{R}}$ is a Lévy process with characteristic function (2.1) and $\mathbb{E}|L(1)|^{r}<\infty$ for some $r>0$, then the stable $\operatorname{CARMA}(p, q)$ process $Y$ as specified in (2.3) exists, if condition (2.6) is satisfied. Then for fixed $0 \leq t_{1}<t_{2}<\cdots<t_{n}$ the cumulant generating function of $\left(Y\left(t_{1}\right), Y\left(t_{2}\right), \ldots, Y\left(t_{n}\right)\right)$ is given by

$$
\begin{aligned}
\ln \mathbb{E} & {\left[e^{i \theta_{1} Y\left(t_{1}\right)+\ldots+i \theta_{n} Y\left(t_{n}\right)}\right] } \\
= & \int_{0}^{\infty} \xi\left(\sum_{j=1}^{n} \theta_{j} \mathbf{b}^{T} e^{A\left(t_{j}+u\right)} \mathbf{1}_{p}\right) d u+\int_{0}^{t_{1}} \xi\left(\sum_{j=1}^{n} \theta_{j} \mathbf{b}^{T} e^{A\left(t_{j}-u\right)} \mathbf{1}_{p}\right) d u \\
& +\int_{t_{1}}^{t_{2}} \xi\left(\sum_{j=2}^{n} \theta_{j} \mathbf{b}^{T} e^{A\left(t_{j}-u\right)} \mathbf{1}_{p}\right) d u+\cdots+\int_{t_{n-1}}^{t_{n}} \xi\left(\theta_{n} \mathbf{b}^{T} e^{A\left(t_{n}-u\right)} \mathbf{1}_{p}\right) d u .
\end{aligned}
$$

In particular, the marginal cumulant generating function is

$$
\ln \mathbb{E}\left[e^{i \theta Y(t)}\right]=\int_{0}^{\infty} \xi\left(\theta \mathbf{b}^{T} e^{A u} \mathbf{1}_{p}\right) d u, \quad t \geq 0 .
$$

Proof. For $j=1, \ldots, n$, use (2.3) and (2.7) to write

$$
\begin{array}{r}
Y\left(t_{j}\right)=\mathbf{b}^{T} \mathbf{X}\left(t_{j}\right)=\mathbf{b}^{T}\left[\int_{(-\infty, 0]} e^{A\left(t_{j}-u\right)} \mathbf{1}_{p} d L(u)\right. \\
+\int_{\left(0, t_{1}\right]} e^{A\left(t_{j}-u\right)} \mathbf{1}_{p} d L(u) \\
\left.+\cdots+\int_{\left(t_{j-1}, t_{j}\right]} e^{A\left(t_{j}-u\right)} \mathbf{1}_{p} d L(u)\right] .
\end{array}
$$

Therefore,

$$
\begin{aligned}
& \theta_{1} Y\left(t_{1}\right)+\cdots+\theta_{n} Y\left(t_{n}\right) \\
& =\int_{(-\infty, 0]} \sum_{j=1}^{n} \theta_{j} \mathbf{b}^{T} e^{A\left(t_{j}-u\right)} \mathbf{1}_{p} d L(u)+\int_{\left(0, t_{1}\right]} \sum_{j=1}^{n} \theta_{j} \mathbf{b}^{T} e^{A\left(t_{j}-u\right)} \mathbf{1}_{p} d L(u) \\
& \quad+\int_{\left(t_{1}, t_{2}\right]} \sum_{j=2}^{n} \theta_{j} \mathbf{b}^{T} e^{A\left(t_{j}-u\right)} \mathbf{1}_{p} d L(u)+\cdots+\int_{\left(t_{n-1}, t_{n}\right]} \theta_{n} \mathbf{b}^{T} e^{A\left(t_{n}-u\right)} \mathbf{1}_{p} d L(u) .
\end{aligned}
$$


Since a Lévy process has the Lebesgue measure as control measure, (2.9) follows now by Proposition 3.4.2 of Samorodnitsky and Taqqu [18].

Throughout, we will assume the following:

\section{Assumption 2.4.}

(1) The driving Lévy process $L$ is $\alpha$-stable for $\alpha \in(0,2)$.

(2) The characteristic polynomials $a(\cdot)$ and $b(\cdot)$ have no common factors, $a(\cdot)$ is nonzero on the imaginary axis and satisfies condition (2.6). All zeros of $a(\cdot)$ are distinct. (3) All component processes of $\mathbf{X}$ and the CARMA process $Y$ are causal and strictly stationary (which is guaranteed by Proposition 2.3).

Proposition 2.5 (cf. Brockwell et al. [5], Proposition 2).

Under Assumption 2.4, the stable $\operatorname{CARMA}(p, q)$ process $Y$ can be represented as a sum of dependent and possibly complex-valued CAR(1) processes:

$$
Y(t)=\sum_{i=1}^{p} Y^{(i)}(t), \quad t \geq 0
$$

where

$$
Y^{(i)}(t)=\kappa_{i} \int_{(-\infty, t]} e^{\lambda_{i}(t-u)} d L(u) \quad \text { and } \quad \kappa_{i}=\frac{b\left(\lambda_{i}\right)}{a^{\prime}\left(\lambda_{i}\right)}, \quad i=1, \ldots, p
$$

and $a^{\prime}(\cdot)$ denotes the derivative of $a(\cdot)$.

Note that the statement of Proposition 2.5 can be extended for the case of eigenvalues of algebraic multiplicity greater than 1, cf. Equations (2)-(4) in Tsai and Chan [20].

Recall that Bernhardt et al. [1] have fitted a stable ARMA process to the Singapore electricity spot price data. Different model selection criteria supported the use of an $\operatorname{ARMA}(1,2)$ or an $\operatorname{ARMA}(2,1)$ model. Consequently, a CARMA(2,1) model should give a good fit to these data. It will be our leading example throughout the paper.

Example 2.6. [Stable $\operatorname{CARMA}(2,1)$ process]

We consider the particular case of a stable $\operatorname{CARMA}(2,1)$ process, which is under Assumption 2.4 the strictly stationary and causal solution of the equations

$$
\left(D^{2}+a_{1} D+a_{2}\right) Y(t)=\left(b_{0}+D\right) D L(t), \quad t \in \mathbb{R},
$$

i.e.

$$
b(z)=b_{0}+z \quad \text { and } \quad a(z)=z^{2}+a_{1} z+a_{2}=\left(z-\lambda_{1}\right)\left(z-\lambda_{2}\right),
$$


where $\lambda_{1} \neq \lambda_{2}$ and both have negative real parts. The kernel of $Y$ is

$$
g(h)=\kappa_{1} e^{\lambda_{1} h}+\kappa_{2} e^{\lambda_{2} h}=\frac{b_{0}+\lambda_{1}}{\lambda_{1}-\lambda_{2}} e^{\lambda_{1} h}+\frac{b_{0}+\lambda_{2}}{\lambda_{2}-\lambda_{1}} e^{\lambda_{2} h} \quad \text { for } h \geq 0 .
$$

In consequence, $Y$ has the representation

$$
Y(t)=\kappa_{1} \int_{(-\infty, t]} e^{\lambda_{1}(t-u)} d L(u)+\kappa_{2} \int_{(-\infty, t]} e^{\lambda_{2}(t-u)} d L(u), \quad t \in \mathbb{R} .
$$

\section{Estimation of the stable CARMA model}

Now we investigate the estimation of stable CARMA models. After deriving some theoretical properties of the sampled process in Section 3.1, we discuss the estimation of the CARMA parameters and the recovery of the driving Lévy process in Sections 3.2 and 3.3, respectively. We assume throughout that Assumption 2.4 holds.

\subsection{Properties of the sampled process}

In the following we assume that the stable CARMA process is observed at the closely and uniformly spaced times $0, h, 2 h, \ldots, N h$, where $h>0$ is prespecified and $N=$ $[T / h]$ is the integer part of $T / h$. Hence inference will be based on the observations $\{Y(n h), n=0, \ldots,[T / h]\}$ of the sampled process. The following result is an $\alpha$-stable version of Lemma 2.1 of Brockwell and Lindner [7], see also Proposition 3 of Brockwell et al. [5]

Proposition 3.1. (a) For given $h>0$ the sampled process $\left\{Y_{n}^{(h)}\right\}_{n \in \mathbb{Z}}:=\{Y(n h)\}_{n \in \mathbb{Z}}$ is given by

$$
Y_{n}^{(h)}=\sum_{i=1}^{p} Y_{n}^{(i, h)}, \quad n \in \mathbb{Z},
$$

where the discrete-time processes $\left\{Y_{n}^{(i, h)}\right\}_{n \in \mathbb{Z}}$ for $i=1, \ldots, p$ are obtained by sampling the component $\operatorname{CAR}(1)$ process $\left\{Y^{(i)}(t)\right\}_{t \in \mathbb{R}}$ as defined in (2.11) at spacing $h$. Since we work with a strictly stationary version of $Y$ we consider

$$
Y_{n}^{(i, h)}=e^{\lambda_{i} h} Y_{n-1}^{(i, h)}+Z_{n}^{(i, h)}, \quad n \in \mathbb{Z},
$$

with noise

$$
Z_{n}^{(i, h)}=\kappa_{i} \int_{((n-1) h, n h]} e^{\lambda_{i}(n h-u)} d L(u), \quad n \in \mathbb{Z}
$$


The rv's $Z_{n}^{(i, h)}$ are $S_{\alpha}\left(c\left|\kappa_{i}\right|\left[\int_{0}^{h} e^{\alpha \lambda_{i}(h-u)} d u\right]^{1 / \alpha}, \beta \operatorname{sign}\left(\kappa_{i}\right), 0\right)$-distributed; for fixed $h, i$, but varying $n$, they are iid; for fixed $h, n$, but varying $i$, they are dependent.

(b) The sampled process $\left\{Y_{n}^{(h)}\right\}_{n \in \mathbb{Z}}$ satisfies the equations

$$
\phi(B) Y_{n}^{(h)}=\prod_{i=1}^{p}\left(1-e^{\lambda_{i} h} B\right) Y_{n}^{(h)}=U_{n}^{(h)}, \quad n \in \mathbb{Z},
$$

where $B$ denotes the backshift operator and

$$
\phi(z)=\prod_{i=1}^{p}\left(1-e^{\lambda_{i} h} z\right)=1-\phi_{1} z-\cdots-\phi_{p} z^{p} .
$$

The process $\left\{U_{n}^{(h)}\right\}_{n \in \mathbb{Z}}$ has representation

$$
U_{n}^{(h)}=W_{n, 0}^{(h)}+W_{n, 1}^{(h)}+\cdots+W_{n, p-1}^{(h)}, \quad n \in \mathbb{Z},
$$

where

$$
W_{n, j}^{(h)}=\sum_{i=1}^{p} \eta_{j}^{(i, h)} Z_{n-j}^{(i, h)}
$$

and the $Z_{n-j}^{(i, h)}$ are as in (3.3). For all $n \in \mathbb{Z}$ the vector $\left(W_{n, 0}^{(h)}, \ldots, W_{n, p-1}^{(h)}\right)$ has independent components, which for $j=0, \ldots, p-1$ are $S_{\alpha}\left(\sigma_{j}^{(h)}, \beta_{j}^{(h)}, 0\right)$-distributed with

$$
\begin{aligned}
& \sigma_{j}^{(h)}=c\left(\int_{0}^{h}\left|f_{j}^{(h)}(u)\right|^{\alpha} d u\right)^{1 / \alpha}, \\
& \beta_{j}^{(h)}=\frac{\int_{0}^{h}\left|f_{j}^{(h)}(u)\right|^{\alpha} \operatorname{sign}\left(f_{j}^{(h)}(u)\right) \beta d u}{\int_{0}^{h}\left|f_{j}^{(h)}(u)\right|^{\alpha} d u},
\end{aligned}
$$

and $f_{j}^{(h)}(u):=\sum_{i=1}^{p} \eta_{j}^{(i, h)} \kappa_{i} e^{\lambda_{i}(h-u)}$ for $0 \leq u \leq h$. The $\eta_{j}^{(i, h)}$ are given in $(3.12)$.

(c) The process $\left\{U_{n}^{(h)}\right\}_{n \in \mathbb{Z}}$ is $(p-1)$-dependent, and for all $n \in \mathbb{Z}$ the rv $U_{n}^{(h)}$ is $S_{\alpha}\left(\sigma_{*}^{(h)}, \beta_{*}^{(h)}, 0\right)$ distributed, where

$$
\begin{aligned}
& \sigma_{*}^{(h)}=\left(\sum_{j=0}^{p-1}\left(\sigma_{j}^{(h)}\right)^{\alpha}\right)^{1 / \alpha}, \\
& \beta_{*}^{(h)}=\frac{\sum_{j=0}^{p-1} \beta_{j}^{(h)}\left(\sigma_{j}^{(h)}\right)^{\alpha}}{\sum_{j=0}^{p-1}\left(\sigma_{j}^{(h)}\right)^{\alpha}},
\end{aligned}
$$

and $\sigma_{j}^{(h)}$ and $\beta_{j}^{(h)}$ are given in (3.7) and (3.8), respectively. 
Proof. (a) Equations (3.2) and (3.3) are obtained by setting $t=n h$ in (2.10) and (2.11). The distribution of $Z_{n}^{(i, h)}$ follows from Samorodnitsky and Taqqu [18], Property 3.2.2. (b) Applying the operator $\prod_{r=1}^{p}\left(1-e^{\lambda_{r} h} B\right)$ to both sides of equation (3.1), we obtain the left hand side of equation (3.4). Now, for the right hand side, observe that

$$
U_{n}^{(h)}=\sum_{i=1}^{p}\left[\left(\prod_{\substack{r=1 \\ r \neq i}}^{p}\left(1-e^{\lambda_{r} h} B\right)\right) Z_{n}^{(i, h)}\right]
$$

and, in consequence,

$$
U_{n}^{(h)}=\sum_{i=1}^{p}\left(\eta_{0}^{(i, h)} Z_{n}^{(i, h)}+\eta_{1}^{(i, h)} Z_{n-1}^{(i, h)}+\cdots+\eta_{p-1}^{(i, h)} Z_{n-p+1}^{(i, h)}\right),
$$

where (we denote by $\sum^{\prime}$ a sum taken over different indices only)

$$
\begin{aligned}
\eta_{0}^{(i, h)} & =1 \\
\eta_{1}^{(i, h)} & =-\sum_{r \neq i} e^{\lambda_{r} h} \\
\eta_{2}^{(i, h)} & =\sum_{r, k \neq i}^{\prime} e^{\left(\lambda_{r}+\lambda_{k}\right) h} \\
\eta_{3}^{(i, h)} & =-\sum_{r, k, j \neq i} e^{\left(\lambda_{r}+\lambda_{k}+\lambda_{j}\right) h} \\
\vdots & \\
\eta_{p-1}^{(i, h)} & =(-1)^{p-1} e^{\sum_{r \neq i} \lambda_{r} h} .
\end{aligned}
$$

Next notice that for all $j=0, \ldots, p-1$, by change of variables and the fact that $L$ has stationary increments, for all $n \in \mathbb{Z}$,

$$
Z_{n}^{(i, h)} \stackrel{d}{=} \kappa_{i} \int_{(0, h]} e^{\lambda_{i}(h-u)} d L(u)
$$

This distribution is independent of $n$, but not independent of $i$ (for $p \geq 2$ ). Moreover, because of Assumption 2.4(2) we have $b\left(\lambda_{i}\right) \neq 0$, hence $\kappa_{i} \neq 0$, so that the random variables $Z_{n-j}^{(i, h)}$ for $i=1, \ldots, p$ are pairwise dependent, cf. Samorodnitsky and Taqqu [18], Theorem 3.5.3. Now, rewrite (3.11) as

$$
U_{n}^{(h)}=\sum_{i=1}^{p} \eta_{0}^{(i, h)} Z_{n}^{(i, h)}+\sum_{i=1}^{p} \eta_{1}^{(i, h)} Z_{n-1}^{(i, h)}+\cdots+\sum_{i=1}^{p} \eta_{p-1}^{(i, h)} Z_{n-p+1}^{(i, h)} .
$$


Define $W_{n, j}^{(h)}:=\sum_{i=1}^{p} \eta_{j}^{(i, h)} Z_{n-j}^{(i, h)}$ for $j=0, \ldots, p-1$ and note that they are independent. Next, observe that (3.6) holds and, according to Samorodnitsky and Taqqu [18], Property 3.2.2, it follows that $W_{n, j}^{(h)} \sim S_{\alpha}\left(\sigma_{j}^{(h)}, \beta_{j}^{(h)}, 0\right)$, where $\sigma_{j}^{(h)}$ and $\beta_{j}^{(h)}$ are as in (3.7) and (3.8).

(c) The $(p-1)$-dependence of $\left\{U_{n}^{(h)}\right\}_{n \in \mathbb{Z}}$ is obvious, whereas the formulas (3.9) and (3.10) follow from [18], Property 1.2.1.

Remark 3.2. (Properties of the vector $\left.\mathbf{Z}_{n}^{(h)}:=\left(Z_{n}^{(1, h)}, \ldots, Z_{n}^{(p, h)}\right)^{T}\right)$

(1) Following Samorodnitsky and Taqqu [18], Prop. 3.4.3, the vector $\mathbf{Z}_{n}^{(h)}$ is $\alpha$ stable, and by [18], Corollary 3.5.4 the components of $\mathbf{Z}_{n}^{(h)}$ are pairwise dependent. Dependence for stable vectors is given by the spectral measure $\Gamma$, a measure on the unit sphere $\mathcal{S}_{p}:=\{\mathbf{x}:\|\mathbf{x}\|=1\} \subseteq \mathbb{R}^{p}$ in $\mathbb{R}^{p}$ and $\|\cdot\|$ is an arbitrary norm in $\mathbb{R}^{d}$. Now define, for $u \in[0, h]$, the vector $\tilde{g}(u):=\left(\kappa_{1} e^{\lambda_{1}(h-u)}, \ldots, \kappa_{p} e^{\lambda_{p}(h-u)}\right)$ and the control measure

$$
m_{1}(d u)=\|\tilde{g}(u)\|^{\alpha} d u
$$

From [18], Prop. 3.4.3, we can derive that for $\alpha \neq 1$ the spectral measure $\Gamma$ is for every Borel set $A \subseteq \mathcal{S}_{p}$ given by

$$
\Gamma(A)=\frac{1+\beta}{2} m_{1}\left(g^{-1}(A)\right)+\frac{1-\beta}{2} m_{1}\left(g^{-1}(-A)\right),
$$

and

$$
g^{-1}(A)=\left\{u \in[0, h]: \frac{\tilde{g}(u)}{\|\tilde{g}(u)\|} \in A\right\} .
$$

Considering the image $\tilde{g}([0, h])$ for $h>0$ and recalling that $\lambda_{i} \neq \lambda_{j}$ for $i \neq j$, we see that $\Gamma$ is not concentrated on a finite number of points on $\mathcal{S}_{p}$ (and hence $\mathbf{Z}_{n}^{(h)}$ cannot be expressed as a linear transformation of a vector of independent $\alpha$-stable random variables, cf. [18], Prop. 2.3.7).

(2) For $\alpha \neq 1$ the characteristic function of the vector $\mathbf{Z}_{n}^{(h)}$ is given by

$$
\begin{aligned}
& \phi\left(\theta_{1}, \ldots, \theta_{p}\right)= \\
& \exp \left\{-\int_{0}^{h}\left|\sum_{j=1}^{p} \theta_{j} \kappa_{j} e^{\lambda_{j}(h-u)}\right|^{\alpha}\left(1-i \beta \operatorname{sign}\left(\sum_{j=1}^{d} \theta_{j} \kappa_{j} e^{\lambda_{j}(h-u)}\right) \tan \frac{\pi \alpha}{2}\right) d u\right\},
\end{aligned}
$$


cf. [18], Prop. 3.4.2. If $\beta=0$, we therefore have

$$
\begin{aligned}
\phi\left(\theta_{1}, \ldots, \theta_{p}\right) & =\exp \left\{-\int_{0}^{h}\left|\sum_{j=1}^{p} \theta_{j} \kappa_{j} e^{\lambda_{j}(h-u)}\right|^{\alpha} d u\right\} \\
& =\exp \left\{-\int_{0}^{h}\left|\sum_{i=1}^{p} \sum_{j=1}^{p} \theta_{i} \theta_{j} \kappa_{i} \kappa_{j} e^{\left(\lambda_{i}+\lambda_{j}\right)(h-u)}\right|^{\alpha / 2} d u\right\} .
\end{aligned}
$$

\subsection{Estimation of the CARMA parameters}

The first inferential goal is to obtain a good estimator $\widehat{\boldsymbol{\Delta}}$ of the CARMA parameter vector $\boldsymbol{\Delta}:=\left(a_{1}, \ldots, a_{p}, b_{0}, \ldots, b_{q-1}\right)$. In the $L^{2}$-case, Brockwell et al. [5] have shown that, for fixed $h$, the mean corrected sampled process $\left\{Y_{n}^{*(h)}:=Y_{n}^{(h)}-E Y_{n}^{(h)}\right\}_{n \in \mathbb{Z}}$ satisfies the $\operatorname{ARMA}(p, q)$ equations, where the innovation process is not necessarily i.i.d. (cf. Proposition 3.1). The proof is based on the fact that every $q$-dependent process in $L^{2}$ has a $\mathrm{MA}(q)$ representation; cf. Proposition 3.2.1 in Brockwell and Davis [4]. For the construction of the corresponding white noise process one uses projections in $L^{2}$, where two zero-mean random variables are orthogonal if and only if they are uncorrelated.

Although for $\alpha<2$ some concepts of orthogonality have been developed, e.g. the James orthogonality as defined in Samorodnitsky and Taqqu [18], Section 2.9, an analogue to Proposition 3.2.1 of Brockwell and Davis [4] does not exist. Moreover, for the definition of an ARMA process with infinite variance innovations, the white noise variables are required to be independent. Consequently, it is not clear how to deal with the $(p-1)$-dependent MA part of (3.4).

Concerning the estimation of the coefficients, we are on safe grounds, at least for ARMA models, since it has been shown that parameter estimation in infinite variance ARMA models may be treated not differently than in a Gaussian ARMA model; cf. Davis [8] and Mikosch et al. [16] and references therein. Indeed, the rate of convergence is for the heavy-tailed stable case better than in the $L^{2}$-case.

Motivated by this, we will treat the cases $\alpha<2$ just like the Gaussian case $\alpha=2$. We want to emphasize that, although theoretical second or higher moments do not exist, their empirical counterparts do. Consequently, on an empirical level we can calculate all necessary quantities.

Firstly, we calculate the same projection as in the $L^{2}$ case. Then we estimate 
the ARMA parameters $\widehat{\boldsymbol{\beta}}=\left(\widehat{\phi}_{1}, \ldots, \widehat{\phi}_{p}, \widehat{\theta}_{1}, \ldots, \widehat{\theta}_{q}\right)$ by a pseudo-maximum likelihood method (alternatively one could also use a least squares method). And, finally, we transform the ARMA parameters into the CARMA parameters $\widehat{\Delta}=\left(\widehat{a}_{1}, \ldots, \widehat{a}_{p}, \widehat{b}_{0}, \ldots, \widehat{b}_{q-1}\right)$.

We start with the left-hand side of (3.4) and remark that the autoregessive coefficients $\phi_{1}, \ldots, \phi_{p}$ depend on the eigenvalues $\lambda_{r}$ for $r=1, \ldots, p$ and on the time $h$ between two subsequent observations. For the right-hand side of (3.4) we first assume a moving average representation for $U_{n}^{(h)}$, ignoring the $(p-1)$-dependence and the fact that we are not in $L^{2}$. Now we estimate the ARMA parameters $\widehat{\boldsymbol{\beta}}=$ $\left(\widehat{\phi}_{1}, \ldots, \widehat{\phi}_{p}, \widehat{\theta}_{1}, \ldots, \widehat{\theta}_{q}\right)$ by maximum likelihood.

To obtain the CARMA parameters we match the left-hand side of (2.2) with the left-hand side of $(3.4)$, which gives the estimates for $\left(a_{1}, \ldots, a_{p}\right)$ from the estimates for $\left(\phi_{1}, \ldots, \phi_{p}\right)$. The estimates for $\left(b_{0}, \ldots, b_{q-1}\right)$ result from a matching argument of the (empirical) autocorrelation function as follows.

Note that the autocorrelations of $U_{n}^{(h)}$ can be calculated using the autocorrelations of the original CARMA process, by calculating the autocorrelations of the process $\phi(B) Y_{n}^{(h)}$. More precisely, due to (3.4) we can write the autocovariances as

$$
\gamma_{U}(k)=\operatorname{cov}\left(\phi(B) Y_{n}^{(h)}, \phi(B) Y_{n-k}^{(h)}\right), \quad k \in \mathbb{Z}
$$

Now, one can plug into (3.15) the exact formula for $\gamma_{Y}(\cdot)$ given in Section 2 of Brockwell et al. [5] as

$$
\gamma_{Y}(k)=2 c^{2} \boldsymbol{b}^{\prime} e^{A|k|} \Sigma \boldsymbol{b}, \quad k \in \mathbb{Z}
$$

where $c$ is the scaling parameter of the stable Lévy process, $A$ and $\boldsymbol{b}$ are as in the state space equations (2.3) and (2.4), and $\Sigma=\int_{0}^{\infty} e^{A y} \boldsymbol{e} \boldsymbol{e}^{\prime} e^{A^{\prime} y} d y$. So finally, we get $\left(b_{0}, \ldots, b_{q-1}\right)$ by matching $\gamma_{U}(k) / \gamma_{U}(0)$ for sufficiently many $k$ with the autocorrelations of a $\mathrm{MA}(q)$ process, using the right-hand side of (3.15), where we have plugged in the ARMA estimates.

We explain this in detail for our relevant example. We emphasize again that the necessary stable theory will be treated elsewhere. In this applied paper we will check whether this approximation is reasonable, and assess the quality of the corresponding estimates in a simulation study in Section 4.

Example 3.3. [Stable CARMA $(2,1)$ process, continuation of Example 2.6] Assume that we have observed a sampled version of the CARMA $(2,1)$ process $\{Y(t), 0 \leq$ 
$t \leq T\}$ with parameters $\boldsymbol{\Delta}=\left(a_{1}, a_{2}, b_{0}\right)$ on a grid with grid size $h>0$ and, based on this, we have estimated the $\operatorname{ARMA}(2,1)$ parameters $\boldsymbol{\beta}=\left(\phi_{1}, \phi_{2}, \theta\right)$. We immediately get from the left-hand side of (3.4)

$$
\phi_{1}=e^{\lambda_{1} h}+e^{\lambda_{2} h}, \quad \phi_{2}=-e^{\left(\lambda_{1}+\lambda_{2}\right) h} .
$$

From $\widehat{\phi}_{1}, \widehat{\phi}_{2}$ we get $\widehat{\lambda}_{1}, \widehat{\lambda}_{2}$ giving $\widehat{a}_{1}=-\left(\widehat{\lambda}_{1}+\widehat{\lambda}_{2}\right)$ and $\widehat{a}_{2}=\widehat{\lambda}_{1} \widehat{\lambda}_{2}$. To obtain an estimator for $b_{0}$ we proceed as described above. To calculate explicit expressions for $\gamma_{U}(0)$ and $\gamma_{U}(1)$, denote the autocorrelation function of $\left\{Y_{n}^{(h)}\right\}_{n \in \mathbb{Z}}$ by $\gamma_{Y}$. We invoke (3.15) to calculate $\gamma_{U}(0)$ and $\gamma_{U}(1)$ as

$$
\begin{aligned}
& \gamma_{U}(0)=\left(1+\phi_{1}^{2}+\phi_{2}^{2}\right) \gamma_{Y}(0)+\left(2 \phi_{1} \phi_{2}-2 \phi_{1}\right) \gamma_{Y}(1)-2 \phi_{2} \gamma_{Y}(2), \\
& \gamma_{U}(1)=-\phi_{2} \gamma_{Y}(3)+\phi_{1}\left(\phi_{2}-1\right) \gamma_{Y}(2)+\left(\phi_{2}^{2}+\phi_{1}^{2}+1-\phi_{2}\right) \gamma_{Y}(1)+\phi_{1}\left(\phi_{2}-1\right) \gamma_{Y}(0) .
\end{aligned}
$$

Recall from Example 3.1.1 of Brockwell and Davis [4] that the autocorrelation function at lag 1 of an arbitrary MA(1) process with coefficient $\theta$ is given by

$$
\frac{\gamma_{U}(1)}{\gamma_{U}(0)}=\frac{\theta}{1+\theta^{2}} .
$$

Now recall from (3.16) the exact formulas for $\gamma_{Y}(\cdot)$, which depend on the CARMA parameters $a_{1}, a_{2}$ and $b_{0}$. To calculate the remaining CARMA parameter $b_{0}$ we plug in the estimates $\widehat{a}_{1}, \widehat{a}_{2}$ into the right-hand side expressions of (3.18). The resulting estimated expressions depend now on $b_{0}$ only, and we plug them into the left-hand side of (3.19). It remains to solve this non-linear equation for $b_{0}$ numerically.

To estimate confidence regions for the parameter vector

$$
\boldsymbol{\Delta}=\left(a_{1}, \ldots, a_{p}, b_{0}, \ldots, b_{q-1}\right)=:\left(\delta_{1}, \ldots, \delta_{p+q}\right)
$$

we use Proposition 4 and Remark 6 in Brockwell et al. [5], which are based on results by Pham [17], Francq and Zakoïan [10] and Marquardt and Stelzer [14] for the $L^{2}$-case. Later, we adapt these confidence regions to the $\alpha$-stable case. Firstly, by Proposition 4 of Brockwell et al. [5] (under a second moment condition), $\widehat{\Delta}$ is strongly consistent for every $h>0$ as $T \rightarrow \infty$. Moreover, a good approximation to the covariance matrix of $\widehat{\Delta}$ (based on the continuous observations of the CARMA process on $[0, T]$ ) is given by $V:=M^{-1} / T$, where $M=\left(M_{k j}\right)_{j, k=1, \ldots, p+q}$ and

$$
M_{j k}=\frac{1}{2 \pi} \int_{-\infty}^{\infty}\left\{\frac{\partial}{\partial \delta_{j}} \frac{a(i \omega)}{b(i \omega)}\right\}\left\{\frac{\partial}{\partial \delta_{k}} \frac{a(-i \omega)}{b(-i \omega)}\right\}\left|\frac{b(i \omega)}{a(i \omega)}\right|^{2} d \omega .
$$


This is due to the weak convergence of $\sqrt{T}\left(\widehat{\Delta}_{\text {MLE }}-\Delta\right)$ to a normal distribution with mean vector 0 and covariance matrix $M^{-1}$ (under the moment condition $\left.E|L(1)|^{4+\delta}<\infty\right)$, where $\widehat{\Delta}_{\text {MLE }}$ denotes a corresponding maximum likelihood estimator in the Gaussian model.

Example 3.4. [Stable CARMA $(2,1)$ process, continuation of Example 3.4] In the CARMA $(2,1)$ case, we have $\delta_{1}=a_{1}, \delta_{2}=a_{2}, \delta_{3}=b_{0}$. We can calculate the elements of $M$ as

$$
\begin{array}{ll}
M_{11}=1 /\left(\sqrt{2}\left(C_{+}+C_{-}\right)\right), & M_{12}=M_{21}=0, \\
M_{22}=1 /\left(2 \sqrt{2} a_{2}^{2}\left(1 / C_{+}+1 / C_{-}\right)\right), & M_{13}=M_{31}=-b_{0} /\left(a_{2}+a_{1} b_{0}+b_{0}^{2}\right), \\
M_{33}=1 /\left(2 b_{0}\right), & M_{23}=M_{32}=1 /\left(a_{2}+a_{1} b_{0}+b_{0}^{2}\right),
\end{array}
$$

where

$$
C_{+}:=\sqrt{a_{1}^{2}+a_{1} C_{0}-2 a_{2}}, \quad C_{-}:=\sqrt{a_{1}^{2}-a_{1} C_{0}-2 a_{2}}, \quad C_{0}:=\sqrt{a_{1}^{2}-4 a_{2}} .
$$

We first report on results for Gaussian CARMA processes. Using the approximating matrix $V=M^{-1} / T$ of the covariance matrix of $\widehat{\Delta}$, one can derive asymptotic confidence regions for $\boldsymbol{\Delta}$, which are valid for a Gaussian CARMA process continuously observed on $[0, T]$. Brockwell et al. [5] have shown in simulations that for general Lévy-driven CARMA processes in $L^{2}$ that there is a close correspondence between the values of $\sqrt{V_{i i}}$ for $i=1, \ldots, p+q$ (i.e. the asymptotic standard deviations of the estimates in $\widehat{\boldsymbol{\Delta}})$ and the sample standard deviations $s\left(\delta_{i}\right)$ of estimates from sufficiently many simulations for $h$ very close to 0 (cf. Brockwell et al. [5], Remark 7).

For $\alpha$-stable CARMA processes $(\alpha<2)$ we expect a similar correspondence between the empirical standard deviations of the estimates for a CARMA process observed on a fine grid at $0, h, 2 h, \ldots,\lfloor T / h\rfloor h$ and the sample standard deviations $s\left(\delta_{i}\right)$ from simulated data also observed at $0, h, 2 h, \ldots,\lfloor T / h\rfloor h$. Furthermore, one can see from simulations, that these sample standard deviations increase with $h$, so that for a discretely observed process the confidence regions derived from $V$ (i.e. as if observed continuously) usually will be too small and, in consequence, one may undervalue the uncertainty about the parameter estimates. Hence, for $h$ not so close to 0 , we adapt the matrix $V$ in order to provide more reliable confidence regions. To this end we define a diagonal matrix $D:=\operatorname{diag}\left(s\left(\delta_{i}\right) / \sqrt{V_{i i}}, i=1, \ldots, p+q\right)$ and adapt $V$ by setting $W:=D V D$. Then the matrix $W$ is a covariance matrix, where the correlations between all components are the same as in $V$, but the standard deviations of the 
components are exactly $s\left(\delta_{i}\right)$. Recall that in the case, where $h$ is small, $s\left(\delta_{i}\right)$ is very close to $\sqrt{V_{i i}}$, and, hence, $W \approx V$.

We shall show this method at work in Section 5.2.

\subsection{Recovering the background driving Lévy process}

In order to suggest a parametric model for the background driving Lévy process, we have to recover the realization of $L$ from our observations, using given or estimated values of $\left(a_{1}, \ldots, a_{p}\right)$ and $\left(b_{0}, \ldots, b_{q-1}\right)$. Following Brockwell et al. [5] one can use the state-space representation of the CARMA process and work with the state vector $\mathbf{X}$ and the canonical state vector $\mathbf{Y}$. Essential for this approach is that the SDE of the $\mathrm{CAR}(1)$ factors in (2.10) given by

$$
d Y^{(i)}(t)+\lambda_{i} Y^{(i)}(t)=\kappa_{i} d L(t)
$$

for $i=1, \ldots, p$ has a pathwise solution. Observe that the Lévy processes in [5] are subordinators, so that the pathwise integral exists obviously. For $\alpha$-stable processes the following has been shown by Fristedt and Taylor [11].

Proposition 3.5. Let $L$ be an $\alpha$-stable Lévy process for $\alpha \in(0,2)$. Assume that $L$ does not have a drift for $\alpha<1$ and that the Lévy measure is symmetric for $\alpha=1$. Then $L$ has finite $p$-variation for $p>\alpha$ and infinite $p$-variation for $p \leq \alpha$.

It has been shown by Young [21] that for such processes a pathwise (RiemannStieltjes) integral exists for appropriate integrands. Moreover, a partial integration rule has been proved. Indeed, for deterministic integrands, and integrators with arbitrary positive $p$ variation, Young's results apply.

Example 3.6. [Stable CARMA $(2,1)$ process, continuation of Example 3.4] In the CARMA $(2,1)$ case, we have $\mathbf{X}(t)=\left(X^{(0)}(t), X^{(1)}(t)\right)$ and $\mathbf{Y}(t)=\left(Y^{(1)}(t), Y^{(2)}(t)\right)$ for $t \geq 0$. Now following equations (2.18) and (5.3)-(5.5) of Brockwell et al. [5] we get 
for $t \geq 0$

$$
\begin{aligned}
X^{(0)}(t) & =X^{(0)}(0) e^{-b_{0} t}+\int_{0}^{t} e^{-b_{0}(t-u)} Y(u) d u \\
X^{(1)}(t) & =-b_{0} X^{(0)}(t)+Y(t) \\
\left(\begin{array}{c}
Y^{(1)}(t) \\
Y^{(2)}(t)
\end{array}\right) & =\frac{1}{\lambda_{1}-\lambda_{2}}\left(\begin{array}{cc}
\lambda_{2}\left(b_{0}+\lambda_{1}\right) & -\left(b_{0}+\lambda_{1}\right) \\
-\lambda_{1}\left(b_{0}+\lambda_{2}\right) & b_{0}+\lambda_{2}
\end{array}\right)\left(\begin{array}{c}
X^{(0)}(t) \\
X^{(1)}(t)
\end{array}\right), \\
L(t) & =\frac{2 \lambda_{r}+a_{1}}{b_{0}+\lambda_{r}}\left[\begin{array}{cc}
\left.Y^{(r)}(t)-Y^{(r)}(0)-\lambda_{r} \int_{0}^{t} Y^{(r)}(s) d s\right], \quad r=1,2 .
\end{array}\right.
\end{aligned}
$$

The last equation represents two different possibilities to recover the driving Lévy process. It is, however, advantageous to take that $r$, where $\lambda_{r}$ has the smallest absolute value (for a reasoning of this choice see Brockwell et al. [5], Section 6).

\section{Simulation study}

We conduct a simulation study to test the estimation procedure for the CARMA parameters and to assess the quality of the estimates. To this end, we simulate sample paths of $\alpha$-stable CARMA $(2,1)$ processes for various values of $\alpha$, and with CARMA parameters $a_{1}=1.9647, a_{2}=0.0893$ and $b_{0}=0.1761$. These are exactly the values we found for the Singapore electricity data, cf. Section 5.

Since the parameters are estimated as if the driving Lévy process was a Brownian motion (which corresponds to the case $\alpha=2$ ), it is particularly interesting to see, how the estimates behave for different values of $\alpha$. Therefore, we repeat the study for five different scenarios, with $\alpha$ taking on the values 2.0, 1.8, 1.6, 1.4 and 1.2302 (cf. Section 5), respectively. The other parameters of the stable distribution of the driving Lévy process are chosen as $\beta=0.1719, c=16.8713$, and $\mu=-4.5388$, so that they correspond to the estimates in the Singapore electricity data.

In each of the five scenarios, we simulate 1000 time series, consisting of 1369 observations each (note that our data set in Section 5 consists of 1369 observations). We choose $h=1$, so that the observation times are $t=1,2, \ldots, 1369$. The CARMA process, however, is sampled for each integer multiple of 0.01, i.e. at times $0.01,0.02,0.03, \ldots, 1369$, using an Euler approximation of the corresponding integrals.

For each realization we computed estimates of the CARMA parameters $a_{1}, a_{2}$ and $b_{0}$ as described in Example 3.3 and then, subsequently for the five different scenarios, 
calculated the mean, bias and the sample standard deviation for the 1000 estimates each. The results are reported in Table 4.1 .

As expected from theory (cf. Davis [8] and Mikosch et al. [16]), the estimates of $a_{1}$ and $a_{2}$ get better, in terms of the sample bias and standard deviation, when $\alpha$ decreases. This effect is much weaker for $b_{0}$. This may be due to the fact that the $(p-1)$-dependent process $\left\{U_{n}^{(h)}\right\}_{n \in \mathbb{Z}}$ has a (weak) MA(1) representation only in the case $\alpha=2$, whereas for $\alpha<2$, the structure of the process $\left\{U_{n}^{(h)}\right\}_{n \in \mathbb{Z}}$ is more complicated as specified in Proposition 3.1.

\begin{tabular}{llccc}
\hline & & $a_{1}$ & $a_{2}$ & $b_{0}$ \\
\hline$\alpha=2.0$ & true & 1.9647 & 0.0893 & 0.1761 \\
\hline & mean & 2.0112 & 0.0975 & 0.1853 \\
$\alpha=1.8$ & bias & 0.0465 & 0.0082 & 0.0092 \\
& std. dev. & 0.1674 & 0.0416 & 0.0462 \\
$\alpha=1.6$ & mean & 2.0021 & 0.0929 & 0.1862 \\
& bias & 0.0374 & 0.0036 & 0.0101 \\
& std. dev. & 0.1533 & 0.0391 & 0.0457 \\
$\alpha=1.4$ & mean & 1.9879 & 0.0956 & 0.1879 \\
& bias & 0.0232 & 0.0063 & 0.0118 \\
& std. dev. & 0.1324 & 0.0371 & 0.0459 \\
& mean & 1.9738 & 0.0927 & 0.1873 \\
& bias & 0.0091 & 0.0034 & 0.0112 \\
& std. dev. & 0.1115 & 0.0359 & 0.0455 \\
& mean & 1.9693 & 0.0912 & 0.1881 \\
& bias & 0.0046 & 0.0019 & 0.0120 \\
& std. dev. & 0.1038 & 0.0323 & 0.0442 \\
\hline
\end{tabular}

Table 4.1: Simulation study for different values of $\alpha$, based on 1000 data sets each: mean, bias, and sample standard deviation of estimates for the CARMA parameters $a_{1}, a_{2}$, and $b_{0}$. 


\section{$5 \quad$ Singapore electricity data}

In this section we fit a stable CARMA $(2,1)$ model to data from the Singapore New Electricity Market. The data can be downloaded at www.ema.gov.sg. It consists of 1369 daily spot prices measured in Singapore Dollars per MegaWatthour (SGD/MWh) and covers the period from January 1, 2005 to September 30, 2008.

Recall that Bernhardt et al. [1] fitted a stable ARMA process to the same data set (with the exception that their data set covered only the period from January 1, 2005 to April 11, 2007). Their model choice was based on the AICC and BIC criteria and showed clearly that either an $\operatorname{ARMA}(1,2)$ or an $\operatorname{ARMA}(2,1)$ model should be used. Every simple AR model led to a much higher AICC and BIC value. Consequently, we expect that a CARMA $(2,1)$ model should give a good fit to these data. Before fitting the CARMA $(2,1)$ model, we first remove trend and seasonal components, which are obviously present in the data.

\subsection{Removing trend and seasonal components}

The original spot price data from the Singapore New Electricity Market is shown in Figure 1. Applying just a trend function for the seasonality shows a rather bad fit, in particular for the first half of 2008. Since energy prices are usually related to the overall inflation rate, we account for this and use also the accumulated inflation rate

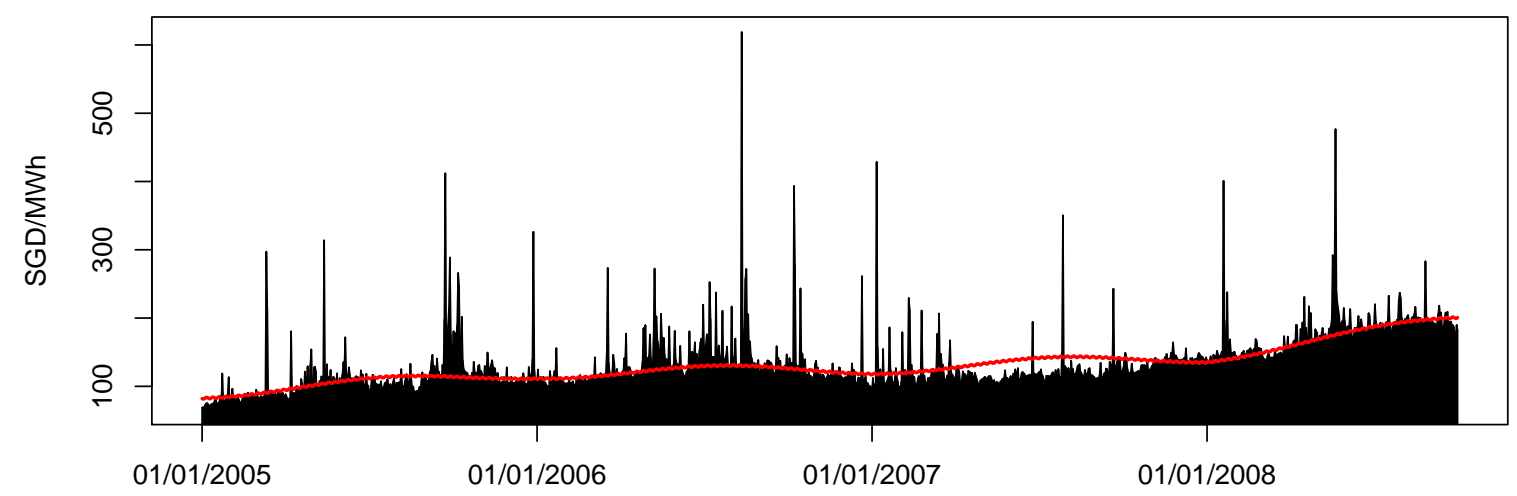

Figure 1: Singapore New Electricity Market: Daily spot prices from January 1, 2005 to September 30, 2008, overlaid by the estimated trend and seasonal curve. 
$r_{t}$ in Singapore from the beginning of our time series up to time $t$ as an exogenous variable for our trend function. Consequently, we fit the function

$$
\Lambda_{t}=\beta_{0}+\beta_{1} \cos \left(\frac{\tau_{1}+2 \pi t}{365}\right)+\beta_{2} \cos \left(\frac{\tau_{2}+2 \pi t}{7}\right)+\beta_{3} r_{t}
$$

to the data. Estimation by a robust least squares method leads to the following parameter estimates:

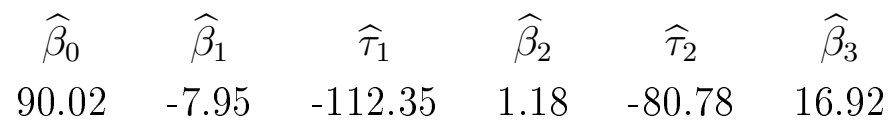

Figure 1 contains the estimated trend and seasonal curve. This is subtracted from the original data and the remaining time series which is shown in Figure 2 is modelled in the following as a CARMA $(2,1)$ process driven by a stable Lévy process. From this plot we expect the innovations of this driving Lévy process to be positively skewed. In Figure 3 one can find the empirical autocorrelation functions of the detrended and deseasonalized series and of its squares. Whereas there are significant autocorrelations up to about lag 20 present in the detrended and deseasonalized data, one does not find any autocorrelation in the squared series.

Answering a question of one of the referees, for our detrended and deseasonalized data, we estimate the Hurst exponent $\widehat{H}=0.8209$ with a standard error of 0.0292 , using Higuchi's [12] method. Accordingly, an approximate symmetric 95\% confidence

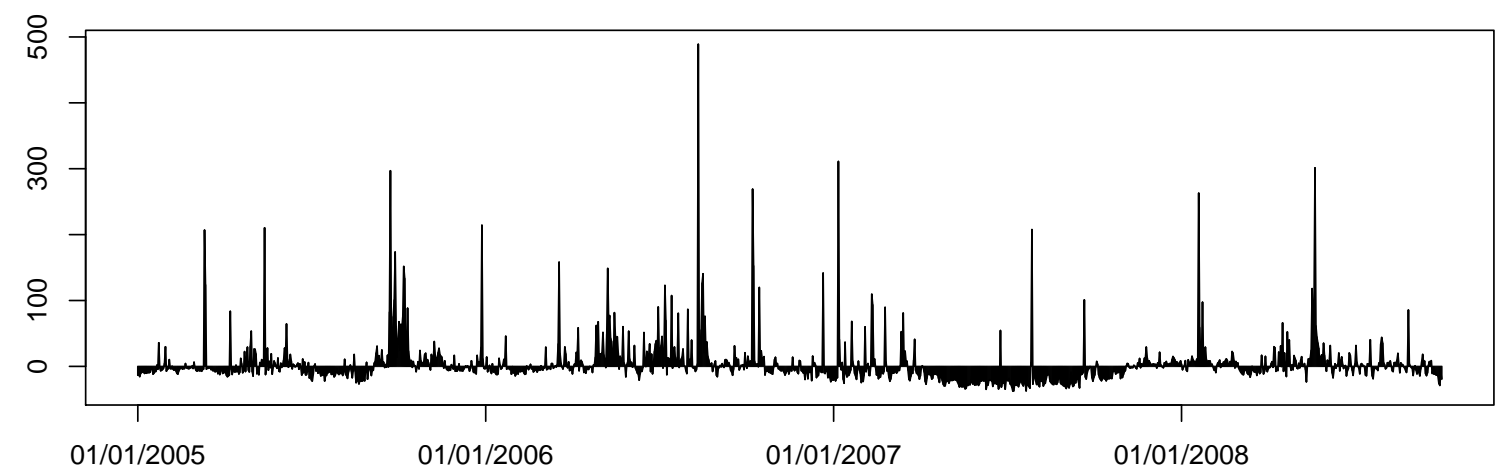

Figure 2: Singapore New Electricity Market: Daily spot prices from January 1, 2005 to September 30, 2008, after removing trend and seasonal components. 

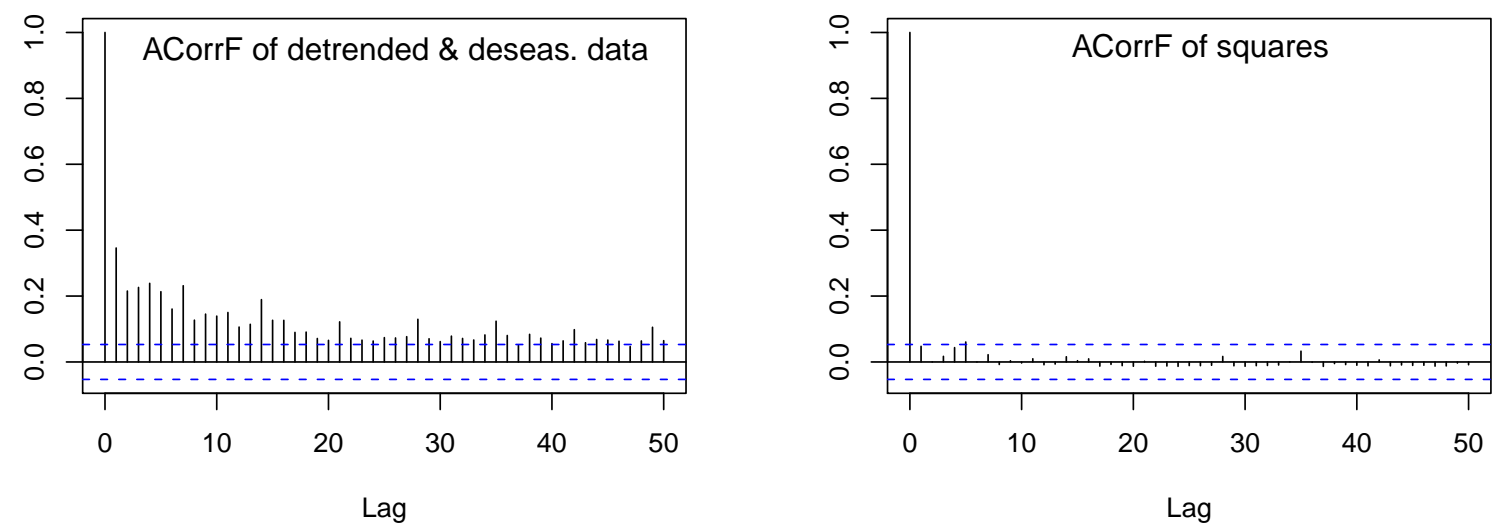

Figure 3: Empirical autocorrelation functions of detrended and deseasonalized series and its squares.

interval for $H$ is given by $[0.7637,0.8781]$. For a finite variance time series, this would indicate that a long range dependence is present in the data, since $\widehat{H}>0.5$. However, here we deal with an infinite variance time series, and according to Samorodnitsky and Taqqu [18], Chapter 7, a long range dependence would be present in our stable setup, if $H-1 / \alpha>0$. As we will see later, in our data we find $\widehat{\alpha}=1.2302$, so that we have $1 / \widehat{\alpha}=1 / 1.2302=0.8129$. Hence, taking the above confidence interval for $H$ into account, $\widehat{H}-1 / \widehat{\alpha}$ is not significantly different from 0 , and we conclude that no long range dependence is present in the detrended and deseasonalized data.

\section{$5.2 \quad$ Fitting the CARMA $(2,1)$ process}

We first estimate the CARMA parameters $a_{1}, a_{2}$, and $b_{0}$, and then try to recover the driving Lévy process. For the estimation of the CARMA parameters we proceed as described in Section 3.2 (and specified in Example 3.3) which leads to the following estimates:

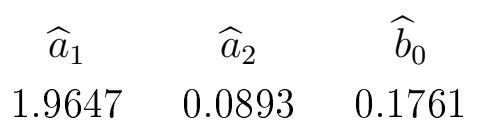

Hence the fitted CARMA $(2,1)$ model is

$$
\left(D^{2}+1.9647 D+0.0893\right) Y(t)=(0.1761+D) D L(t), \quad t \in[0,1369],
$$


with estimated autoregressive roots $\widehat{\lambda}_{1}=-0.0465$ and $\widehat{\lambda}_{2}=-1.9181$. Note that the estimated model is stationary by Proposition 2.2. To get reliable confidence regions for the parameter estimates we apply the method of Section 3.2. The asymptotic covariance matrix $V=M^{-1} / 1369$ can be calculated by plugging the estimates $\widehat{a}_{1}, \widehat{a}_{2}$, and $\widehat{b}_{0}$ into the formulas for $M_{j k}$ as calculated in Example 3.4. This yields

$$
V=10^{-4} \times\left[\begin{array}{rrr}
52.8907 & 12.2657 & 16.2995 \\
12.2657 & 8.7831 & 8.2655 \\
16.2995 & 8.2655 & 10.9837
\end{array}\right]
$$

and the preliminary standard deviations $\sqrt{V_{11}}=0.07273, \sqrt{V_{22}}=0.02964, \sqrt{V_{33}}=$ 0.03314 , for the estimates $\widehat{a}_{1}, \widehat{a}_{2}$, and $\widehat{b}_{0}$, respectively. As argued in Section 3.2, the confidence regions derived from $V$ may be too small due to the discretization. On the other hand, we want to emphasize that confidence regions in stable discrete time linear models are smaller than in the $L^{2}$-case. Nonetheless, to be on the safe side, we use the estimated sample standard deviations from the simulation study in Section 4 for comparison. There we found, for $\alpha=1.2302$ (cf. below) the sample standard deviations $\widehat{s}\left(a_{1}\right)=0.1038, \widehat{s}\left(a_{2}\right)=0.0323, \widehat{s}\left(b_{0}\right)=0.0442$, for $\widehat{a}_{1}$, $\widehat{a}_{2}$, and $\widehat{b}_{0}$ respectively. By setting $D=\operatorname{diag}(0.1038 / 0.07273,0.0323 / 0.02964,0.0442 / 0.02964)$ we get for $W=D V D$

$$
W=10^{-4} \times\left[\begin{array}{rrr}
107.7325 & 19.0766 & 34.6899 \\
19.0766 & 10.4302 & 13.4318 \\
34.6899 & 13.4318 & 24.4252
\end{array}\right] .
$$

Taking this as the corrected covariance matrix, we can now derive an approximate 95\% confidence region. Figure 4 shows three sections through a corresponding $95 \%$ confidence ellipsoid where one of the parameters is fixed to the estimated value each. Note that 0 is not contained in either ellipsoid; i.e. all coefficients are significant.

To get a complete model specification we now also estimate the Lévy increments as described in Section 3.3 in order to specify an appropriate driving Lévy process. The estimates of the Lévy increments $L(n)-L(n-1)$ were obtained by constructing a realization of the component $\mathrm{CAR}(1)$ process corresponding to the estimated autoregressive root $\widehat{\lambda}_{1}$, since this root has a smaller absolute value than $\widehat{\lambda}_{2}$; cf. Example 3.6. The estimated Lévy increments are shown in the first plot of Figure 5. One can see the positive skewness of the innovations. The second row of this plot contains empirical autocorrelation functions of the increments and their squares. Obviously, 

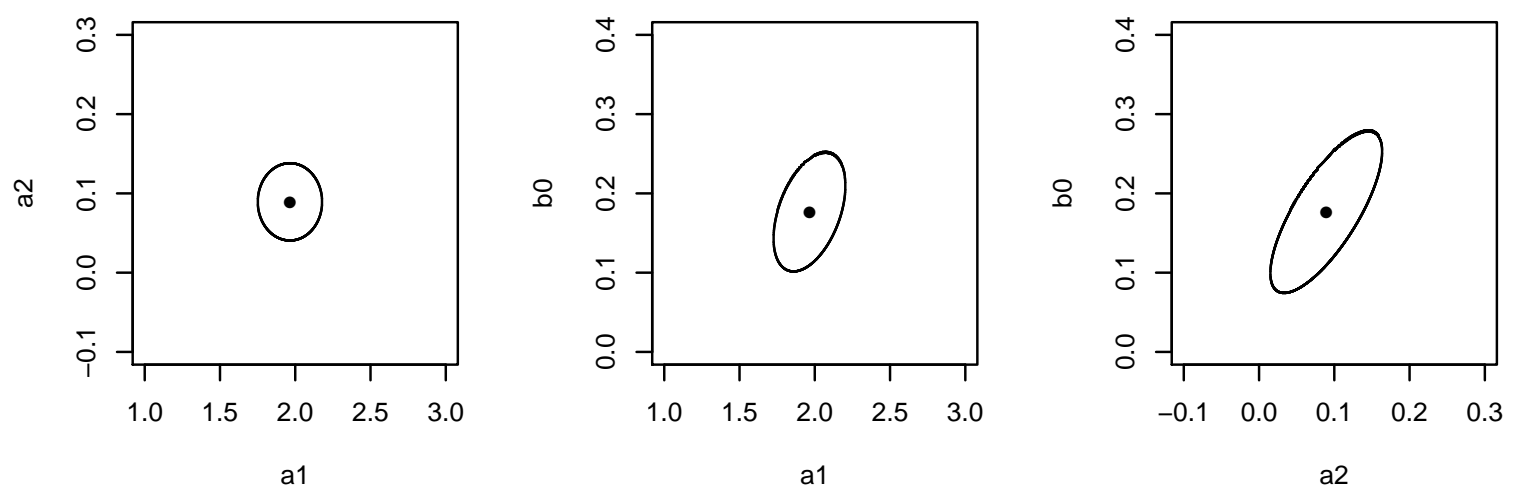

Figure 4: Sections through a $95 \%$ confidence ellipsoid with $b_{0}=0.1761$ fixed (left), $a_{2}=0.0893$ fixed (middle), and $a_{1}=1.9647$ fixed (right). The dots indicate the location of the estimate $\left(\widehat{a}_{1}, \widehat{a}_{2}, \widehat{b}_{0}\right)$.

the dependence structure of the data is well described by our CARMA(2,1) model. Moreover, we can obtain the empirical distribution function for the Lévy increments and a histogram and the empirical distribution function are shown in the last plot of Figure 5 as solid line. Finally we fit a stable distribution to the Lévy increments using the program STABLE. This software is available from J. P. Nolan's website academic2.american.edu/ jpnolan. For our estimated Lévy innovations, it gives the following parameter estimates of the stable distribution:

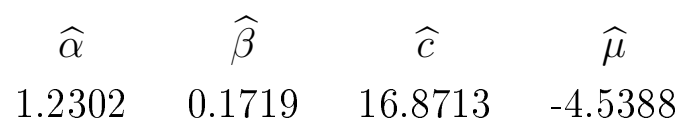

First note, that the estimate of the location parameter $\mu$ is quite small compared to the estimate of the scale parameter $c$. As expected, the estimate $\widehat{\beta}$ of the skewness parameter is positive. Moreover, the STABLE software provides estimated $95 \%$ confidence intervals for $\alpha$ and $\beta$. In our case, these are given by $[1.1522,1.3082]$ and $[0.0595,0.2843]$, respectively. Hence they confirm that the innovations are far from being Gaussian, and that their skewness is significantly different from 0 . The dashed line in the bottom plots of Figure 5 corresponds to the cumulative stable distribution function with these parameters, indicating the excellent fit of the stable distribution to the Lévy increments. 


\section{Summary and conclusion}

In this paper we investigated CARMA processes, which are driven by a stable nonGaussian Lévy process. In particular, we discussed the existence of strictly stationary and causal solutions and derived the cumulant generating function. Using a representation of the stable CARMA $(p, q)$ process as sum of dependent CAR $(1)$ processes we can discribe the sampled CARMA process by an equation, where the left-hand side corresponds to an $\operatorname{AR}(p)$ process and the right-hand side to a $(p-1)$-dependent process. Although, for a stable CARMA process, there is no weak MA representation of this $(p-1)$-dependent process, we have seen in a simulation study, that we can apply the same principle as in the case, where the innovations of the driving Lévy process have finite second moment: the standard deviations of the estimates even decrease as the stable parameter $\alpha$ decreases. Finally, as an application, we fitted a $\operatorname{CARMA}(2,1)$ model to spot prices from the Singapore New Electricity Market.

One of the advantages of continuous-time modelling is that it allows to investigate pricing problems. Hence, we see an interesting future project in using the framework of stable CARMA processes to develop strategies for pricing energy derivatives.

\section{Acknowledgement}

This project was started, when the first author was staying at the Technische Universität München. This visit was supported by the Advance Mathematical Methods for Finance (AMaMeF) grant number 1836 from the European Science Foundation. The revision was done while the last two authors had a very pleasant visit to the Wolfgang Pauli Institute in Vienna.

\section{References}

[1] Bernhardt, C., Klüppelberg C. and Meyer-Brandis, T. (2008) Estimating high quantiles for electricity prices by stable linear models. J. Energy Markets $\mathbf{1}$, No. 1, 3-19.

[2] Brockwell, P. J. (2009) Lévy-driven continuous-time ARMA processes. In: Andersen, T.G., Davis, R.A., Kreiss, J.-P. and Mikosch, T. (Eds.) Handbook of Financial Time Series. Springer, Heidelberg, pp. 653-667. 
[3] Brockwell, P. J. (2001) Lévy-driven CARMA processes. Ann. Inst. Statist. Math. 53, No. 1, 113-124.

[4] Brockwell, P. J. and Davis, R. A. (1991) Time Series: Theory and Methods, 2nd ed. Springer, New York.

[5] Brockwell, P. J., Davis R. A. and Yang, Y. (2009) Estimation for non-negative Lévy-driven CARMA processes. Submitted for publication.

[6] Brockwell, P. J., Hyndman, R. J. and Grunwald, G. K. (1991) Continuous Time Threshold Autoregressive Models. Stat. Sinica 1, No. 2, 401-410.

[7] Brockwell, P.J. and Lindner, A. (2008) Existence and uniqueness of stationary Lévy-driven CARMA processes. Preprint. Technische Universität Braunschweig.

[8] Davis, R. A. (1996) Gauss-Newton and M-estimation for ARMA processes with infinite variance. Stoch. Proc. Appl. 63, 75-95.

[9] Doob, J. L. (1944) The elementary Gaussian processes. Ann. Math. Statist. 25, 229-282.

[10] Francq, C. and Zakoïan, J.-M. (1998) Estimating linear representations of nonlinear processes. J. Stat. Plan. Inference 68, 145-165.

[11] Fristedt, B. and Taylor, S.J. (1973) Strong variation for the sample functions of a stable process. Duke Math. J. 40, 259-278.

[12] Higuchi, T. (1988) Approach to an irregular time series on the basis of the fractal theory. Physica D 31, 277-283.

[13] Klüppelberg, C., Meyer-Brandis, T. and Schmidt, A. (2008) Electricity spot price modelling with a view towards extreme spike risk. Quantitative Finance, to appear.

[14] Marquardt, T. and Stelzer, R. (2007) Multivariate CARMA processes. Stoch. Proc. Appl. 117, 96-120.

[15] Meyer-Brandis, T. and Tankov, P. (2008) Multi-factor jump-diffusion models of electricity prices. IJTAF 11, No. 5, 503-528. 
[16] Mikosch, T., Gadrich, T., Klüppelberg, C. and Adler, R. J. (1995) Parameter estimation for ARMA models with infinite variance innovations. Ann. Stat. 23, 305-326.

[17] Pham, D. T. (1977) Estimation of parameters of a continuous-time Gaussian stationary process with rational spectral density function. Biometrika 64, 385399.

[18] Samorodnitsky, G. and Taqqu, M. S. (1994) Stable Non-Gaussian Random Processes. Chapman and Hall, London.

[19] Tsai, H. and Chan, K. S. (2005) Temporal aggregation of stationary and nonstationary continuous-time processes. Scand. J. Stat. 32, 583-597.

[20] Tsai, H. and Chan, K. S. (2009) A note on the non-negativity of continuous-time ARMA and GARCH processes. Stat. Comput. 19, 149-153.

[21] Young, L.C. (1936) an inequality of Hölder type, connected with Stieltjes integration. Acta Mathematica 67, 251-282.

[22] Weron, R. (2006) Modeling and Forecasting Electricity Loads and Prices: A Statistical Approach. Wiley, Chichester. 

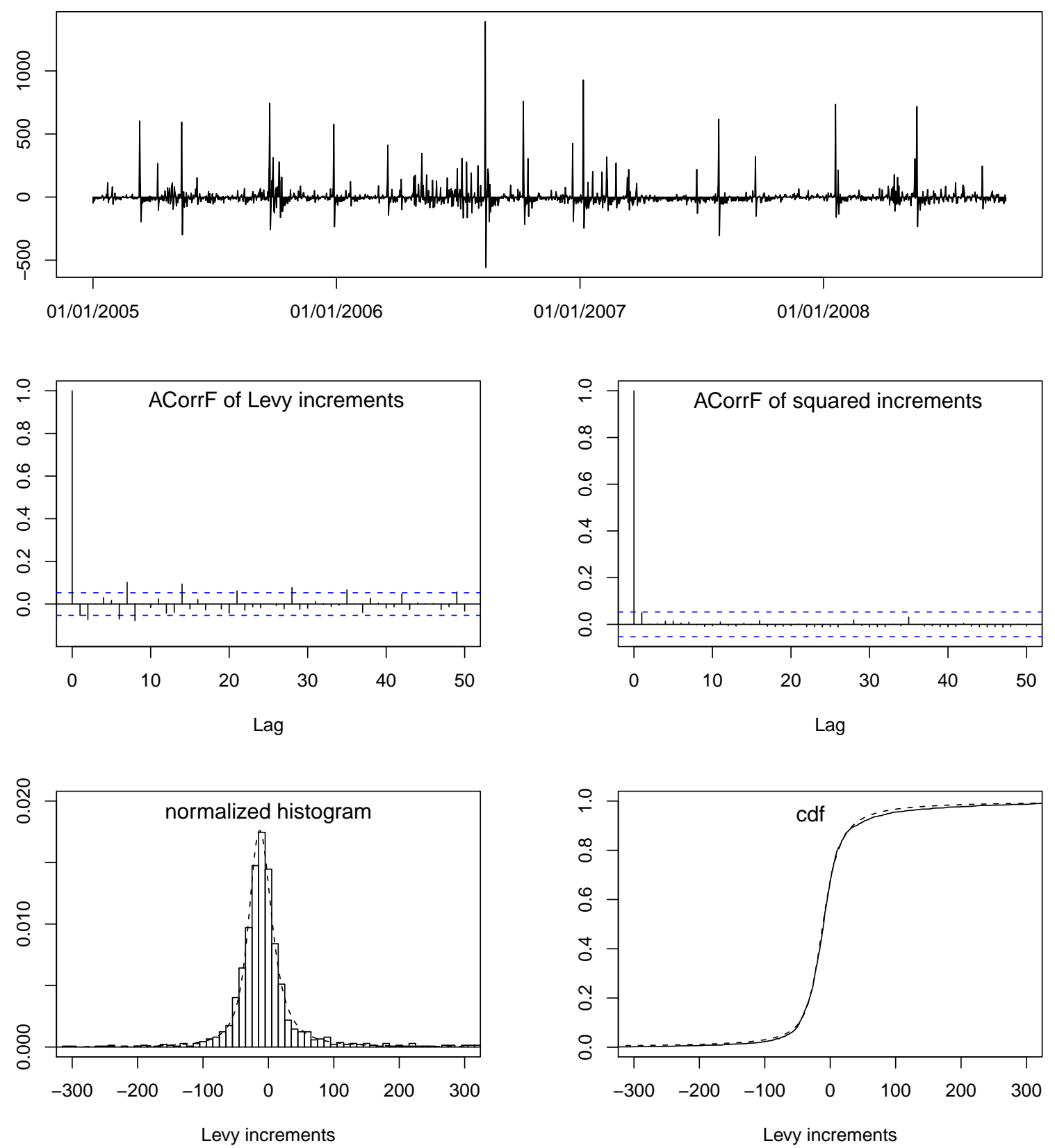

Figure 5: Top: Estimated Lévy increments. Middle: Empirical autocorrelation functions of estimated Lévy increments and squared increments. Bottom: Histogram and empirical distribution function of estimated Lévy increments (solid line) and cumulative distribution function of fitted stable distribution (dashed line). 